\title{
Segregation of cognitive and motor aspects of visual function using induced motion
}

\author{
BRUCE BRIDGEMAN, MARC KIRCH, and ALAN SPERLING \\ University of California, Santa Cruz, California 95064
}

\begin{abstract}
Targets were displaced to cancel an apparent displacement induced by a step motion of a background or were held stationary while appearing to jump in an induced displacement. Target and background were then extinguished, and the subject pointed to the target's last position. When the target had appeared to move but did not, background position did not significantly affect pointing; when the target had moved but appeared to remain stationary (displacement canceled by opposite induced displacement), pointing depended upon the target's egocentric position. A similar result was obtained with sinusoidal motion. In terms of a twovisual-systems hypothesis, the motor system uses more veridical spatial information and is less affected by relative changes in two retinal signals than is the cognitive system.
\end{abstract}

An increase in threshold for detecting target displacements during saccadic eye movements is now well established. First described qualitatively by Ditchburn (1955) and by Wallach and Lewis (1965) and rediscovered independently by Brune and Lücking (1969), the effect has been analyzed more recently by several groups (Beeler, 1967; Mack, 1970; Mack, Fendrich, \& Pleune, 1978). Bridgeman, Hendry, and Stark (1975) showed that the temporal course of the rise in threshold paralleled the saccadic suppression that had been observed for other visual functions and interpreted the effect as a saccadic suppression of displacements. The suppression is quite large, amounting to about $20 \%$ of the magnitude of a saccade, and it appears even when criterion-free measures are used (Bridgeman \& Stark, 1979). The effect is also known to be scalar rather than vectorial in its nature (Bridgeman \& Stark, 1979; Mack, 1970; Stark, Kong, Schwartz, Hendry, \& Bridgeman, 1976).

The saccadic suppression of displacement poses a theoretical difficulty for the maintenance of visual orientation across saccadic eye movements, for it implies a degradation of information about positions of objects in the world after saccades. This interpretation contradicts the common observations that humans have no difficulty in visual-motor coordination despite numerous saccades and that the world remains subjectively stable as well (position constancy).

The two conflicting observations (saccadic suppression of displacement, on the one hand, despite lack of disorientation, on the other) were combined into a single experiment by asking subjects to point to the position of a target that had been displaced and then extinguished (Bridgeman \& Lewis, 1976; Bridgeman, Lewis, Heit, \& Nagle, 1979). When pointing behavior following a detected displacement was compared with pointing following an undetected (suppressed) displacement, it was found that motor orientation was accurate and identical under the two conditions. Failure to detect a displacement had no effect on the accuracy of pointing to the target's new position. Control experiments showed that the effect could not be explained by criterion effects. The result was interpreted in terms of the "two visual systems" hypothesis that has found support in both animals (Held, 1968; Schneider, 1967; Trevarthen, 1968) and humans (Weiskrantz, Warrington, Sanders, \& Marshall, 1974). Interpreted in this context, the saccadic suppression phase of the pointing experiment assessed only a "cognitive" component of the visual system ("focal" in Trevarthen's terminology), while the pointing behavior was driven by the "motor" component ("ambient" to Trevarthen). In these psychophysical experiments, no assumptions could be made about the anatomical locations or physiological mechanisms of the two systems; the systems were defined in terms of response measures, so that the cognitive system was examined with nonisomorphic, symbolic responses, while the motor component was measured with pointing tasks in which the response is isomorphic with stimulus position.

If two psychophysically separable visual systems are functioning in normal humans, it is important to know the degree of linkage between them. The pointing and saccadic suppression experiment outlined above shows only that signals that do not reach the cognitive system, because they are blocked by saccadic suppression of displacement, can still influence motor behavior. From these experiments alone, it would still be possible to interpret the cognitive function as assessing some subset of the information available to a single cognitive and motor system, rather than interpreting the two systems as independent or quasi-independent. A rigorous test of the degree of independence of the cognitive and motor systems would require one condition in which a signal entered 
the cognitive, but not the motor, system, and another in which a signal entered only the motor system. Such a design, in which one condition includes B and not $A$, while the other includes $A$ and not $B$, is necessary to show a double dissociation.

We address this issue by including conditions in which a signal might enter the cognitive system without affecting the motor system. In the saccadic suppression of displacement experiments a retinal displacement signal (the position of the target) was masked cognitively by an extraretinal signal (the corollary discharge accompanying the saccade). This resulted in the suppression of the cognitive component of the perception of position change, but in a preservation of the motor component. Alternatively, one can mask a retinal displacement with another retinal displacement; by changing the conditions appropriately, signals can be selectively shunted to the ambient system alone, the focal system alone, or both.

When a large background texture is displaced abruptly during visual fixation, a smaller target superimposed on it seems to jump in the opposite direction with a smaller amplitude. The effect is analogous to the induced motion obtained with slowly moving backgrounds, but is different in that the abrupt displacement is always visible to the subject. Induced motion of a small target can be obtained either with or without perception of the motion of the inducing background, while induced displacement always entails a perceived background displacement.

Duncker (1929) provided the first thorough descriptions of the effects, giving the same name ("Induzierte Bewegung"') to both of them. They will be separated here for purposes of clarity.

If egocentric calibration is the concern mainly of the motor system, that system should be less sensitive to induced displacement so that its information about egocentric localization will not be falsely biased. By comparing the cognitive appearance of targets in induced displacement with pointing behavior to the same targets after they are extinguished, we can compare the information available to the two systems.

A hypothesis that the cognitive and motor visual systems receive independent information would predict that, when faced with the choice of pointing toward an apparent position or an egocentric position, the subject's pointing would correspond to the egocentric position, while the perceived amount of induced displacement would follow the apparent position.

\section{METHOD}

\section{Apparatus}

Subjects sat before a hemicylindrical screen on which a random dot pattern, $22 \mathrm{deg}$ wide $\times 15 \mathrm{deg}$ high, was projected through a galvanic mirror. A black center square, $1 \mathrm{deg}$ in size, served as a fixation point. The induced target was an inverted isosceles triangle, $1.3 \mathrm{deg}$ wide $\times 1.8 \mathrm{deg}$ high, projected through a second galvanic mirror. The vertical axes of both mirrors were near the axis of the hemicylindrical screen, and the center of the right eye was also near this axis, to minimize distortions resulting from movements of the target and background. The brightness of the screen was $32 \mathrm{~cd} / \mathrm{m}^{2}$, and the brightness of the random dot background was $306 \mathrm{~cd} / \mathrm{m}^{2}$.

\section{Experiment 1: Step Displacements}

The subject was instructed to visually track the center of the square in the background, using saccadic eye movements. In this way, an identical eye movement pattern could be maintained under all experimental and control conditions, despite variations in the direction or magnitude of target displacements. The subjects could not infer target displacements by monitoring the fact that they were making saccades. It was important that the eyes remain fixed during the displacements themselves, however, so that saccadic suppression or extraretinal signals could not affect the results. To avoid this possibility, the subjects were cautioned not to try to anticipate the displacement of the background square, and trials on which the eye anticipated the displacement were discarded. In practice, hardly any of these occurred. Under these conditions, the latency from target displacement to the beginning of the tracking saccade was $150-220 \mathrm{msec}$, long enough to remain beyond the time interval of saccadic suppression of displacement (Bridgeman et al., 1975).

Saccadic tracking enhances the induced-displacement effect, in agreement with Duncker's (1929) observation that when two stimuli are moving relative to one another, the fixated stimulus appears to be moving less, regardless of the relative sizes of the two stimuli. This is true both for step displacements and for sinusoidal movements. The effect does not depend on eye movements themselves because the eyes are fixating during the movements; a more likely explanation is that the eye movements redirect attention.

Horizontal eye movements were monitored with paired infrared sensitive photocells (Bahill, Clark, \& Stark, 1975), a system that allows the use of naive subjects because the apparatus does not contact the eye. Bandwidth of the system is $0-500 \mathrm{~Hz}$.

The experiment was conducted in two successive stages: a calibration in which the subject estimated the amount of cognitive induced displacement by a cancellation technique, and a pointing stage in which the subject pointed to the position of the target. The extent of perceived displacement was first determined by moving the background horizontally in a square-wave pattern generated by a function generator, with an excursion of $14 \mathrm{deg}$ and a period of $1.66 \mathrm{sec} / \mathrm{cycle}$. The rotation of the mirror from one extreme to the other required about $5 \mathrm{msec}$. The superimposed target triangle jumped in the same phase as the background motion, with an amplitude that the subject could vary from 0 to the full extent of the background excursion.

The amount of displacement induced in the cognitive system was assessed with a method of adjustment, in which the subject continuously varied the amplitude of the target jump until the target seemed to be standing still. After a short warm-up period, the subjects made three estimates of the degree of displacement, and the median setting was taken as that subject's cognitive induced displacement. This perception did not change during subsequent steps.

In the second part of the experiment, which followed immediately after the determination of the amount of induced displacement, the target moved with one of three patterns while the subject fixated the center square of the background stimulus, which was always moved in the same manner as in the first phase. In the first condition, the target was moved in the same phase as the background $(t)$, so that the subject experienced no displacement of the target since its induced displacement exactly canceled its egocentric displacement. In the second condition, the target was egocentrically stationary (0), but appeared to undergo an induced displacement with a phase opposite to that of the background. In a third condition, the target moved with the same amplitude as in the first condition, but in the opposite phase (-), so that induced and egocentric displacements added together.

A trial began when the target and background appeared simultaneously and when the subject began tracking the background. After 
about two full cycles of displacement, the experimenter extinguished both the target and the background simultaneously by closing electronically controlled shutters on the respective projectors. This constituted a signal for the subject to move an unseen pointer to a position directly under the downward pointing tip of the target triangle at the time of its disappearance. The subject was instructed to move the pointer-a long rod with its axis at the center of curvature of the screen-from a peripheral position past the position of the triangle and back again to the correct position. The pointer was mounted on a potentiometer, which in turn was connected to a simple circuit that produced a voltage linearly related to the pointer position. This position was then read on an oscilloscope by the experimenter, the pointer moved back to its peripheral position, and the trial concluded. For each trial, the target and background stimuli were extinguished while the target was in one of the extreme positions and after the tracking saccade had been completed. This occurred when the background was on either the left or right side, so that each relative displacement condition could be subdivided into two pointing conditions. The six experimental conditions will be identified as in-phase $(+)$ left and right $(+\mathrm{L}$ and $+\mathrm{R})$, induced displacement without egocentric motion ( 0 ) left and right (OL and $0 \mathrm{R})$, and opposite-phase (-) displacement left and right ( $-\mathrm{L}$ and $-\mathrm{R})$. Left and right were always defined in terms of background position.

Thus, the subject was asked to point to the target under three kinds of conditions: one entailing egocentric displacement but no apparent (cognitive) change in position $(+R$ and $+L)$, a second having apparent displacement but no egocentric motion $(0 R$ and $O L)$, and a third having both egocentric and apparent displacement $(-R$ and $-L)$. The dependent variables were the averages of the pointing determinations in all trials under each condition. The $-R$ and $-L$ conditions, in which the two motions add, were included as controls so that the subjects would experience a variety of displacements and were not analyzed statistically.

The subjects were three paid undergraduate volunteers, all naive about the purposes of the experiment. Each was tested for a total of 360 trials, divided among the six conditions according to a random number table. The subjects were run in three blocks of 120 trials each. One subject was replaced after showing pointing behavior so variable that it frequently exceeded the \pm 10 -deg linear range of our pointer.

\section{Experiment 2: Sinusoidal Motion}

This experiment was identical to the first, except that both target and background underwent sinusoidal motion rather than squarewave displacement. This condition leads to induced motion (Duncker, 1929), in which a large texture moving in the background induces an apparent motion in an egocentrically fixed target of smaller size. Induced motion is interpreted in this context as a trick that the cognitive system uses to gain very high sensitivity to motions of objects in the world by using a relative-motion cue, at the expense of an ambiguity in egocentric localization (Brosgole, 1967, 1968). This view is consistent with the finding that induced motion can be obtained dichoptically (Bassili \& Farber, 1977), and thus probably has a cortical basis.

The term "induced motion" is applied here in its classic definition, indicating any apparent motion of a target induced by the motion of a surrounding frame, even if the motion of the frame is also visible and even if the subject is tracking the moving background. Stimulus parameters were identical to those in Experiment 1, except that the period of a cycle was increased to $9.5 \mathrm{sec}$ $(.105 \mathrm{~Hz})$.

Pilot experiments showed that induced motion is strongest when the target stimulus is superimposed upon a moving background, rather than being surrounded by a moving frame, and when the induced target is not fixated. With this stimulus arrangement, convincing induced motion can be obtained even when motion is too fast to obtain the effect with conventional target-and-frame configurations.

To keep conditions in the sinusoidal motion experiment as close as possible to those in the step displacement experiment, and to keep eye movements the same in all conditions, subjects tracked the background square. Thus, it is logically possible that the Filehne illusion and underregistration of pursuit eye movements might contribute to the motion illusion in this experiment. Equalization of eye movements in all conditions, however, assures that any differences in results between conditions cannot be ascribed to differences in eye movements. After about two full cycles of movement, eye movements in all of the subjects (monitored on an oscilloscope) showed saccade-free sinusoidal pursuit tracking in most trials. Saccade-free predictive pursuit is not difficult at slow pursuit rates, and was learned during training trials. Target and background were extinguished at the extreme of their deviation, when target, background, and tracking eye were all stationary.

The subjects were three additional paid undergraduate volunteers, naive about the purposes of the experiment.

\section{RESULTS}

All of the subjects in both experiments experienced induced displacement or motion ranging from 1.9 to 2.9 deg peak to peak, in the direction predicted from the classical literature. In both experiments, a two-way analysis of variance ( 3 subjects by 4 conditions) showed significant treatment effects but no significant subjects effects [Experiment 1: $F$ (subjects) = $.186, \mathrm{p}=.83, \mathrm{~F}$ (treatments) $=7.31, \mathrm{p}=.0002$; Experiment 2: $\mathrm{F}$ (subjects) $=.312, \mathrm{p}=.74, \mathrm{~F}$ (treatments) $=$ $4.59, \mathrm{p}=.004]$. For further analysis, the results for the subjects in each experiment were pooled, and pointing differences were assessed with a series of orthogonal planned comparisons.

\section{Experiment 1: Step Displacement}

The goal of the experiment, to assess a condition in which information enters the cognitive system but might not enter the motor system, is met most directly in the " 0 " condition. Here the target stimulus is undergoing several degrees of apparent displacement, while its egocentric position remains unvarying. The pointing behavior followed the egocentric position more closely than it followed the apparent position: The difference between pointing when the target was induced to appear on the left and to appear on the right was $16 \mathrm{~min}$, a difference that is not significant at the .05 level $(z=1.93, p>.05)$. Since the direction of motor bias is opposite to that predicted from cognitive induced displacement, it cannot be interpreted as a residual induced effect. The result is summarized in Figure 1 (upper left).

The inverse signal, in which egocentric motion is not accompanied by apparent motion, is obtained in the " + " condition. Here, pointing to the targets on the left was differentiated from pointing to targets on the right by more than 1 deg (Figure 1 , lower left), a statistically significant difference $(z=8.09, p<.001)$. A null hypothesis that subjects would point to the apparent position of the target can thus be rejected. However, pointing was also significantly different from the egocentric position of the target $(z=7.81$, 

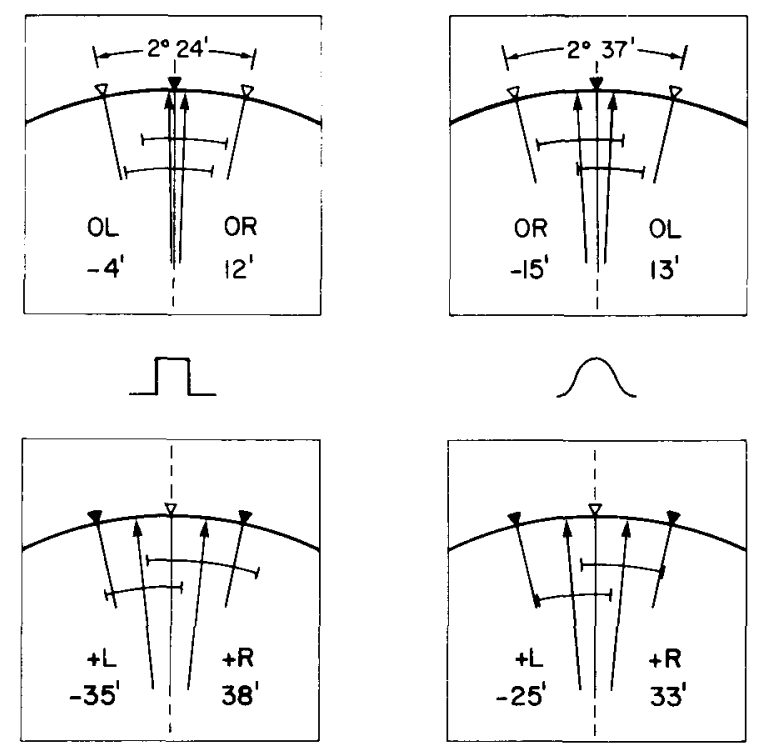

Figure 1. Averaged pointing behavior in "+" and " 0 " conditions. Open triangles represent the apparent position of the target triangle, averaged across subjects, while solid triangles represent the egocentric position of the target triangle under each condition. In the " 0 " conditions (top), the open triangles represent the apparent position of the target at the extremes of its range, while in the "+" conditions (bottom), the solid triangles represent the extremes of egocentric motion. Arrows represent the mean direction of pointing for three subjects in all trials under the condition indicated; mean deviation from the centerline is indicated in minutes of arc below the label for each arrow. Left: Results of Experiment 1 , step displacements. In both " 0 " and " + " conditions, pointing is biased in the directions of the filled (egocentric) triangles. Right: In Experiment 2, sinusoidal motion, the tendency to point toward egocentric position rather than apparent position is less pronounced. In the " 0 " condition, the illustration implies a perceived motion equal to the amount of motion needed to cancel the induced motion in the "+" condition, an assumption that was not tested directly. All angles are exaggerated tenfold for purposes of illustration. Ares = 1 SD.

$\mathrm{p}<.001$ ), indicating a tendency for subjects to point closer to the center of the screen than to the actual position of the target. Thus, the result is intermediate between the hypothesis of independence of information in the two visual systems, which would predict unbiased pointing toward the egocentric location, and the alternative hypothesis that induced displacement influences a unified visual system so that apparent position and pointing behavior would coincide.

In the "-" condition, in which egocentric and induced displacements would be expected to add, subjects also showed a tendency to point closer to the center than to the true position of the targets. Thus, the subjects in this experiment revealed a strong tendency to point closer to the center line than to the true location of the targets, whether induced displacement amplified or eliminated the apparent motion of the target.

\section{Experiment 2: Sinusoidal Motion}

The results of this experiment are similar to those obtained for step displacement, except that differences in pointing in the " 0 " condition were significant. The average pointing on both left and right sides was less than $15 \mathrm{~min}$ arc from the egocentric location of the target, and was less than .5 standard deviation units from the center. The difference between average pointing when the inducing stimulus was on the left and when it was on the right was small but statistically significant $(z=3.74, p<.001)$. Its small magnitude implies that the bias has no functional significance (Figure 1, upper right).

In the " + " condition (Figure 1, lower right), in which the egocentric motion is canceled by induced motion, the pointing behavior becomes significantly different for the two target positions $(z=8.25, \mathrm{p}<$ $.001)$. Again, however, pointing is still significantly closer to the center than to the true egocentric positions of the targets $(\mathrm{z}=13.9, \mathrm{p}<.001)$.

These results were obtained while the subjects underwent significant illusions of motion. All three of them spontaneously and independently reported an impression that motion of the background was being manipulated, although background motion was in fact the same in all conditions.

Despite the difference in the " 0 " condition between the step and the sinusoidal experiments, a twoway analysis of variance (4 target motion conditions, $\mathrm{OL}, \mathrm{OR},+\mathrm{L},+\mathrm{R}$, by 2 motion conditions, step and sinusoidal) shows no significant overall difference between subjects' behavior in the two experiments $(\mathrm{F}=.041, \mathrm{p}=.83)$. This test is not orthogonal to the statistical tests given above and is included only to give an impression of the difference between the two motion conditions.

\section{DISCUSSION}

In these experiments, cognitive or perceived position of a target was contrasted with its egocentric position measured in the motor system by a pointing procedure while the target underwent either induced displacement or a cancellation of induced displacement. In general, the illusions affected pointing less than they affected perceptual experience.

Several effects might have differentiated behavior in the step and the sinusoidal experiments. The first is the Filehne illusion (Stoper, 1967), an apparent motion of a stable background in a direction opposite to pursuit eye movement. The illusion may be related to relative-motion cues during pursuit. The Filehne illusion might be expected to increase the magnitude of the illusion by adding to the induced motion effect. In the square-wave experiment, saccadic eye tracking and steady fixation during target displacements would eliminate the Filehne illusion. 
A second potential cause of difference between the two experiments is the apparent underregistration of pursuit eye movements that has been quantified by several groups (Festinger \& Easton, 1974; Festinger, Sedgwick, \& Holtzman, 1976; Mack \& Herman, 1972; Stoper, 1973). Under some conditions, the underregistration can lead to a loss of position constancy during pursuit (Mack \& Herman, 1973, 1978). During pursuit tracking, illusions of movement occur that imply that the visual system takes into account only a fraction of the actual velocity of pursuit eye movements. Again, the phenomenon would not affect the saccadic tracking of the step displacement condition.

Despite the possible influences that might differentiate pointing behavior in the two conditions, there was no statistically significant difference between the results of the two experiments. The results in the step experiment were closer to those predicted theoretically, but the overall difference between the two experiments was not large enough to be significant, showing that the Filehne illusion and underregistration of pursuit movements were not important influences on the present results. Furthermore, the similarity of illusion magnitude in the saccadic and pursuit conditions shows that the pursuit eye movements themselves were not responsible for the illusion in the second experiment, although they may have contributed to the small degradation in performance.

Another characteristic of pointing behavior common to both experiments was the tendency of all subjects to point closer to the center of the screen than to the egocentric position of the target, when the target was not itself on the midline. The same phenomenon was noted in earlier work that measured pointing to off-center targets (Bridgeman et al., 1979). The explanation for this behavior may be related to the fact that subjects were always pointing to a blank field after the targets and backgrounds had been extinguished. Under a similar condition, in which an inducing frame was occluded following induced motion of a stimulus into the periphery, Brosgole (1967) found that the apparent position of the target drifted back toward the phenomenally straight-ahead position. He termed this effect "induced autokinesis." In the context of the present experiments, induced autokinesis might have moved the apparent position of the target back toward the midline during the interval between the extinguishing of the stimuli and the positioning of the pointer. This interpretation requires that induced autokinesis apply to the motor system as well as to the cognitive system. One significant difference between the present experiments and Brosgole's is that our target disappeared with the background, while Brosgole's target remained visible. In our experiment, we infer an induced autokinesis of the mental image of the stimulus.

In Brosgole's experiment, the inducing frame required $31 \mathrm{sec}$ to drift to its extreme position, and the amount of induced autokinesis was found to equal the amount of drift after a further delay of $31 \mathrm{sec}$. The dynamics of the phenomenon were not assessed, although the target was reported to drift slowly back toward the center. Because our subjects judged the target position after only a few seconds of delay that were needed to adjust the pointer, induced autokinesis would compensate for only a fraction of the eccentricity of the target, in agreement with our results.

Induced autokinesis may itself be an example of a more general effect occurring whenever apparent position conflicts with the corollary discharge (Sperry, 1950) that registers the intended eye position. Matin, Picoult, Stevens, Edwards, Young, and MacArthur (1981) have recently shown such an effect, in which corollary discharge and eye position were separated by the attempt of a partly paralyzed subject to look in an eccentric direction. As long as no attempts at further movement were made, the world was perceived as normal and in the veridical position, an effect that Matin calls visual capture. When the structured visual field was darkened, however, luminous points on which the subject fixated seemed to drift in the direction of the deviated corollary discharge signal and remain there. In Brosgole's experiments, the target appeared off-center because of visual capture by a moving frame of reference, even though the target was still projected on the fovea and the corollary discharge pointed straight-ahead. After the background was extinguished, only the corollary discharge remained to indicate target position, and it gradually came to dominate the position remembered from the inducing frame.

Induced autokinesis and the pointing errors in the present experiments can now be explained with the same mechanism. Visual capture means that, while the corollary discharge is ignored when a visual frame of reference is available, it determines perception when it is the only available indicator of egocentric position. The role of corollary discharge in behavior is seen most clearly in the " + " condition, in which pointing is biased by an egocentric motion even though visual capture prevents perception of the target's deviations.

In the "+" condition, induced autokinesis would have the effect of biasing pointing away from the egocentric position and toward the center of the screen. This is the pattern of results observed in both experiments: In the step experiment, the offset of the pointing direction from the center averaged $51 \%$ of the extent of egocentric motion, while in the sinusoidal experiment, it averaged $37 \%$. In the " 0 " condition, induced autokinesis would be expected to have little effect because pointing is already near the center. Thus, the phenomenon of induced autokinesis, coupled with a hypothesis that subjects will point to the egocentric position rather than to the apparent position, explains 
all of the qualitative results. The bias of pointing toward the center in the "-." condition can also be explained by induced autokinesis.

The general conclusion of the experiment, after considering the above complications in interpretation, is that pointing generally follows egocentric position when egocentric and apparent positions are dissociated. The result was obtained both in the " + " condition, in which a signal entered the motor system without influencing the cognitive system, and in the " 0 " condition, in which a cognitive signal had little influence on the motor system. The cognitive and motor systems clearly assess different systems of spatial organization, although the possibility remains that there is some crosstalk between them.

Several recent studies, using a variety of methods, support this conclusion. Ballistic motor acts (striking a target with a hammer) are accurate even under conditions in which significant illusions of position occur (Hansen, 1979; Hansen \& Skavenski, 1977). The deviations of mean pointing direction from veridicality are even smaller with ballistic pointing than they are in the present experiment, with a pointing task that allows time for proprioceptive feedback; the reports of trial-to-trial variability of the response are more difficult to interpret, however, because Hansen and Skavenski report SE (or standard error), a measure of the likely deviation of the mean of the next $\mathbf{N}$ trials from the present sample mean, rather than SD (or standard deviation), a measure of the likely deviation of the next trial from the mean. SE can be decreased by increasing the number of trials, making interpretation in terms of physiological variables more difficult.

In a recent study, similar to the step displacement portion of the present study, but using a different response measure, Wong and Mack (1980) found that saccadic tracking eye movement is generally in the accurate direction, even when induced displacement makes the target appear to move in the opposite direction. In their study, the target always appeared to move. Mack et al. (1978) demonstrated a result similar to the sinusoidal experiment's " 0 " condition by showing lack of eye tracking to a retinally stabilized target undergoing induced motion.

Miller (1980), however, draws a contradictory conclusion in a series of careful studies comparing motor and perceptual measures during saccades and pursuit eye movements, showing that a perceptual and a motor (saccade) measure yield small mean errors (less than $2 \mathrm{deg}$ ), but that the motor measure shows a smaller estimate of movement magnitude than the perceptual measure under all conditions, and the pursuit system suffers from greater underestimates of motion than does the saccadic system. This contradiction of the present result may allow a finer differentiation of efferent systems, for it is known that there are signif- icant differences between information available to the eye movement control system and the skeletal musculature (arm pointing). Ono and Nakazimo (1977) have shown that the eye and the pointing motor systems reach different conclusions about the positions of targets following vergences. Thus, the motor system as introduced above may itself have components with access to different kinds of spatial information.

The sinusoidal motion experiment is similar to a recent induced motion study by Farber (1979), with one methodological exception. In his experiment, subjects were simultaneously viewing and pointing to a moving target, so that cognitive and motor activities were taking place simultaneously, and a conflict might arise between the two visual systems: If a subject perceives a target as standing still under conditions in which a real motion cancels an induced motion, he would feel it inconsistent to move a pointer to track the target even if the motor system were receiving more egocentrically accurate information. This is another example of visual capture by a structured visual field, and may explain why Farber reports that "relative motion" is the most important determiner of active tracking (pointing) while the present study had the opposite result. We eliminated the conflicting information by extinguishing the target before pointing. In the language of the two-visualsystems interpretation, Farber has shown that the cognitive system dominates when the two systems receive simultaneous contradictory information.

Our study shows that the motor system's output can be isolated from the cognitive system by eliminating all image information during the motor pointing procedure, forcing the subject to rely on only his internal map of visual space to guide pointing. When the cognitive system is subject to illusions of induced motion, this procedure shows that contradictory and more accurate spatial information is retained in a separate map of visual space, a map that is used by the motor system to guide behavior but is not normally accessible to experience.

Information can be routed independently to the cognitive or motor visual systems, so that a change in the information present in one of the systems need not significantly influence the information in the other. The result is shown most clearly in Experiment 1 , possibly because of the lack of pursuit eye movements. Independence of the two systems cannot be explained as selective access of the two output modes to a single topographic representation of visual space, is not a "disconnection syndrome" created by experimental or natural lesions, and extends to spatial perception and motor coordination as well as to saccadic eye movement control. Future studies of visuomotor coordination must take the independence of cognitive and motor functions into account. 


\section{REFERENCES}

Bahill, A. T., Clark, M. E., \& Stark, L. Dynamic overshoot in saccadic exe movements is caused by neurological control signal reversals. Experimental Neurology, 1975, 48, 95-112.

Bassili, J. N., \& Farber, J. M. Experiments on the locus of induced motion. Perception \& Psychophysics, 1977, 21, 157-161.

BEELER, G. Visual threshold changes resulting from spontaneous saccadic eye movements. Vision Research, 1967, 7, 769-775.

Bridgeman, B., Hendry, D., \& Stark, L. Failure to detect displacement of the visual world during saccadic eye movements. Vision Research, 1975, 15, 719-722.

Bridgeman, B., \& Lewis, S. How the eye knows where the world is. Neuroscience Abstracts, 1976, 25, 277.

Bridgeman, B., Lewis, S., Heit, G., \& Nagle, M. The relationship between cognitive and motor-oriented systems of visual position perception. Journal of Experimental Psychology: Human Perception and Performance, 1979, 5, 692-700.

Bridgeman, B., \& Stark, L. Omnidirectional increase in threshold for image shifts during saccadic eye movements. Perception \& Psychophysics, 1979, 25, 241-243.

Brosgole, L. Induced autokinesis. Perception \& Psychophysics, 1967, 2, 69-73.

Brosgole, L. An analysis of induced motion. Acta Psychologica, 1968, 28, 1-44.

Brune, F., \& Lücking, C. H. Oculomotorik, Bewegungswahrnehmung und Raumkonstanz der Seedinge. Der Nervenarzt, 1969, 40, 413-421.

Ditchbunn, R. Eye-movements in relation to retinal action. Optice Acta, 1955, 1, 171-176.

DUNCKER, K. Über induzierte bewegung (Ein beitrag zur Theorie optisch wahrgenommener Bewegung). Psychologische Forschung, 1929, 12, 180-259.

FARBER, J. Manual tracking of induced object motion. Investigative Ophthalmology Supplement, 1979, 18, 3.

Festinger, L., \& Easton, A. Inferences about the efferent system based on a perceptual illusion produced by eye movements. Psychological Review, 1974, 81, 44-58.

Festinger, L., Sedgewick, H., \& Holtzman, J. Visual perception during smooth pursuit eye movements. Vision Research, 1976, 16, 1377-1386.

Hansen, R. Spatial localization during pursuit eye movements. Vision Research, 1979, 19, 1213-1221.

Hansen, R., \& Skavenski, A. Accuracy of eye position information for motor control. Vision Research, 1977, 17, 919-926.

HELD, R. Dissociation of visual functions by deprivation and rearrangement. Psychologische Forschung, 1968, 31, 338-348.

MAck, A. An investigation of the relationship between eye and retinal image movement in the perception of movement. Perception \& Psychophysics, 1970, 8, 291-298.

Mack, A., Fendrich, R., \& Pleune, J. Adaptation to an altered relation between retinal image displacements and saccadic eye movements. Vision Research, 1978, 18, 1321-1327.

Mack, A., \& Herman, E. A new illusion: The underestimation of distance during pursuit eye movements. Perception \& Psychophysics, 1972, 12, 471-473.

Mack, A., \& Herman, E. Position constancy during pursuit eye movements: An investigation of the Filehne illusion. Quarterly Journal of Experimental Psychology, 1973, 25, 71-84.

Mack, A., \& Herman, E. The loss of position constancy during pursuit eye movements. Vision Research, 1978, 18, 55-62.

Matin, L., Picoult, E., Stevens, J., Edwards, M., Young, D., \& MacArthur, R. Visual-context dependent mislocalizations under curare-induced partial paralysis of the extraocular muscles. Investigative Ophthalmology and Visual Science Supplement, 1980, 19, 81.

Mille R, J. Information used by the perceptual and oculomotor systems regarding the amplitude of saccadic and pursuit eye movements. Vision Research, 1980, 20, 59-68.

Ono, H., \& NaKazimo, S. Saccadic eye movements during changes in fixation to stimuli at different distances. Vision Research, 1977, 17, 233-238.

Schneider, G. E. Contrasting visuomotor functions of tectum and cortex in golden hamster. Psychologische Forschung, 1967, 31, 52-68.

Sperry, R. Neural basis of spontaneous optokinetic response produced by visual inversion. Journal of Comparative and Physiological Psychology, 1950, 43, 482-489.

Stark, L., Kong, R., Schwartz, S., Hendry, D., \& Bridgeman, B. Saccadic suppression of image displacement. Vision Research, 1976, 16, 1185-1187.

Stoper, A. Vision during pursuit movement: The role of oculomotor information. Unpublished doctoral dissertation, Brandeis University, 1967.

STOPER, A. Apparent motion of stimuli presented stroboscopically during pursuit movement of the eye. Perception \& Psychophysics, 1973, 13, 201-211.

Trevarthen, C. B. Two mechanisms of vision in primates. Psychologische Forschung, 1968, 31, 299-337.

Wallach, H., \& LEwis, C. The effect of abnormal displacement of the retinal image during eye movements. Perception \& Psychophysics, 1965, 1, 25-29.

Weiskrantz, L., Warrington, D. K., Sanders, M. D., \& Marshall, J. Visual capacity in the hemianopic field following restricted occipital ablation. Brain, 1974, 97, 709-729.

Wong, E., \& MACK, A. Does perceived or retinal locus determine the programming of saccades? Investigative Ophthalmology and Visual Science Supplement, 1980, 19, 81.

(Received for publication August 29, 1980; accepted January 30, 1981.) 